\title{
In vivo targeted mutagenesis of a regulatory element required for positioning the Hoxd-11 and Hoxd-10 expression boundaries
}

\author{
Matthieu Gérard, ${ }^{1}$ Jia-Yang Chen, ${ }^{2}$ Hinrich Gronemeyer, ${ }^{2}$ Pierre Chambon, ${ }^{2}$ Denis Duboule, ${ }^{1,3}$ and \\ József Zákány ${ }^{1}$ \\ ${ }^{1}$ Department of Zoology and Animal Biology, University of Geneva, Sciences III, 1211 Geneva 4, Switzerland; ${ }^{2}$ Institut de \\ Génétique et de Biologie Moléculaire et Cellulaire, CNRS/INSERM/ULP, 67404 Illkirch Cédex, C.U. de Strasbourg, France
}

\begin{abstract}
Vertebrate Hox genes are required for the proper organization of structures along the rostrocaudal axis. Hoxd-11 is expressed in the posterior part of the embryo, up to the level of prevertebra 27, and its expression boundary is reproduced by a Hoxd-11/lacZ transgene. Expression of this transgene anterior to prevertebra 27 is prevented by the silencing activity of a cis-acting element, region IX. Using transgenic mice, we show that Hoxd-11 repression by region IX is necessary to position the sacrum properly. This silencing activity depends on phylogenetically conserved sequences able to bind in vitro retinoic acid receptors and COUP-TFs. ES cells were used to generate mice carrying a subtle mutation that abolishes binding of nuclear receptors to region IX. Mutant mice display an anterior shift of their lumbosacral transition inherited as a codominant trait. In mutant embryos, expression of both Hoxd-11 and Hoxd-10 mRNAs in the prevertebral column is anteriorized. These results illustrate the sharing, in cis, of a single regulatory element in order to establish the expression boundaries of two neighboring Hoxd genes.
\end{abstract}

[Key Words: ES cells; mouse development; HoxD complex; regulatory mutation; nuclear receptors]

Received June 10, 1996; revised version accepted July 23, 1996.

In vertebrates, specific combinations of Hox gene products determine the nature of the structures that appear along the anteroposterior axis (e.g., Kessel and Gruss 1991). Vertebrate Hox genes are organized in four complexes, which appeared during evolution by successive duplications of a unique ancestral complex (e.g., Holland 1992). Hox genes are regulated tightly at the transcriptional level during embryogenesis, and their expression domain along the rostrocaudal axis of the embryo is colinear with their position in the complex, such that $5^{\prime}$ located genes are expressed in more posterior areas. Experiments involving ectopic expression of Hox genes led to abnormal body patterning (Krumlauf 1994), thereby confirming the requirement for a precise control of $H o x$ gene expression during development. The analysis of Hox gene regulation in transgenic mice has revealed that expression patterns can sometimes be recapitulated upon random integration in the genome. However, in many cases transgene expression domains do not fully reproduce the corresponding Hox mRNA accumulation.

${ }^{3}$ Corresponding author.
For instance, the expression domains of Hoxd genes in developing fetal limbs could never be reproduced correctly (e.g., Renucci et al. 1992; Gérard et al. 1993), suggesting that multiple elements, long-range interactions, as well as sharing of regulatory elements between genes are essential for normal in situ regulation (Krumlauf 1994; Van der Hoeven et al. 1996).

We have used the ES cell route (Capecchi 1989) to investigate the role of a Hox regulatory element in the context of the HoxD cluster. Previous studies had identified a series of regulatory elements required to establish the Hoxd-11 mRNA expression boundary in the prevertebral column (Gérard et al. 1993). Hoxd-11, one of the five $A b d B$ related genes of the $\operatorname{Hox} D$ complex, is expressed in the posterior part of the embryo (IzpisúaBelmonte et al. 1991). The rostral expression limit of an Hoxd-11/lacZ transgene was found to be restricted to the level of the pv27 by a negative regulatory element, region IX. Deletion of region IX, which contains five motifs reminiscent of the nuclear receptor binding half site consensus sequence (NRRE), led to anteriorized transgene expression in pv26 and pv25 (Gérard et al. 1993). Here we show that repression of Hoxd-11 is required to 
prevent an anterior shift of the lumbosacral transition. Furthermore, we demonstrate that the NRREs are able to bind, in vitro, members of the nuclear receptor superfamily. A 7-bp mutation that abolished the binding of these nuclear receptors led to anteriorized reporter gene expression in pv25-26 in transgenic mice. To demonstrate the physiological relevance of these results, we generated, via homologous recombination in ES cells, mice carrying the same 7 -bp regulatory mutation in region IX. In such mice, both Hoxd-11 and Hoxd-10 expression boundaries were anteriorized. This engineered mouse Hox regulatory mutant, therefore, illustrates that a single element can regulate in cis the expression of two neighboring genes in the context of the $\operatorname{Hox} D$ cluster.

\section{Results}

Repression of Hoxd-11 is necessary for positioning the sacrum

Expression of Hoxd-11 along the anteroposterior axis of the embryo was largely reproduced by a $7.5-\mathrm{kb}$ reporter gene construct in transgenic mice (Ns-E/lacZ, Fig. 1). The anterior boundaries of both Hoxd-11 mRNA and $1 a c Z$ expression were at the level of pv27 (Fig. 2A). A negative regulatory element, region IX, had been identified previously and localized 3' to the Hoxd-11 transcription unit (Fig. 1; Gérard et al. 1993). Deletion of this element (Ns-H/lac Z construct, Fig. 1) resulted in an anteriorized lacZ expression, with a novel boundary at the level of pv25 (Fig. 2B). To test the importance of Hoxd-11 repression in the pv25-26 domain in vivo, we produced transgenic animals that expressed the genuine Hoxd-11 gene in the context of the Ns-H genomic fragment (NsH/Hoxd-11, Fig. 1), such that Hoxd-11 was expressed ectopically in pv25 and pv26 (Fig. 2; cf. A and B). Wholemount in situ hybridization showed both an anteriorized expression domain and an elevated Hoxd-11 mRNA content in transgenic embryos, as expected from the expression of the Ns-H/lacZ transgene (Fig. 2C,D). Most of the

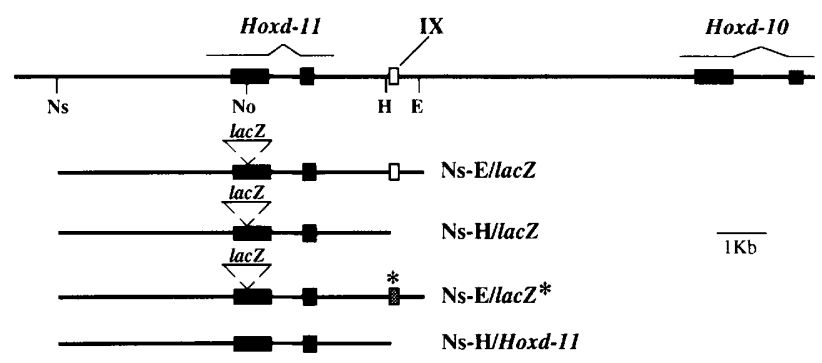

Figure 1. Partial restriction map of the murine Hoxd-11 locus. The position of region IX is shown. Below, the composition of the different transgenes is indicated. Ns-E/lacZ corresponds to the full Hoxd-11 transgene, Ns-H/lacZ is deleted for region IX, and Ns-H/Hoxd-11 is the Hoxd-11 expressing construct without region IX. The Ns-E/lacZ* reporter gene contains the same genomic region as $\mathrm{Ns}-\mathrm{E} / \mathrm{lacZ}$, but with the $\mathrm{C} 6$ mutation present in region IX, as indicated by $\left({ }^{*}\right)$. (Ns) NsiI; (No) NotI; (H) HindIII; (E) EcoRI. nontransgenic littermates displayed a normal pattern of six lumbar vertebrae (L6), whereas transgenic animals showed only five (L5) or even four (L4, in one-third of the cases) lumbar vertebrae (Fig. 2E,F and Table 1). In L4, L5, and L6 animals, the sacral vertebrae were followed posteriorly by six, five, or four caudal vertebrae bearing neural arches, respectively. Therefore, a redistribution of the lumbar versus upper caudal vertebral identities had occurred, and the sacrum apparently shifted as a compact unit of four vertebrae. This led to the presence of 24 or 25 presacral vertebrae, instead of the normal 26, but left the total number of vertebrae with neural arches unchanged (34).

Repression is mediated by a nuclear receptor response element

Sequencing of the Hoxd-11 locus in mouse, birds (Gérard et al. 1993), and fish (M. Gérard and D. Duboule, unpubl.) showed that region IX was well conserved throughout vertebrate species. The mouse element was $89 \%$ identical to its avian counterpart, and a clearly recognizable cognate sequence was found in the zebrafish Hoxd-11 locus, at a similar position. Sequence comparisons further revealed five motifs reminiscent of the nuclear receptor binding half-site consensus sequence (PuGGTCA; Leid et al. 1992), whose positions and orientations were conserved in the three species (Fig. 3A; data not shown).

We assayed for the capacity of both RAR/RXR heterodimers and ARP-1, which belongs to the COUP-TF family (Ladias and Karathanasis 1991), to bind to these motifs by electrophoretic mobility shift assays (EMSA). These experiments revealed that these nuclear receptors were able to bind efficiently to region IX (Fig. 3). EMSA with a series of mutated oligonucleotides indicated that three PuGGTCA-related motifs, organized as an inverted repeat (IR0), as well as one overlapping direct repeat spaced by one base pair (DR1), contributed to the binding of RAR/RXR in vitro (Fig. 3A,B). These binding sites were, therefore, considered to form a potential nuclear receptor response element (NRRE). Binding of RAR/RXR was abolished when all three motifs were mutated (C6 mutation; Fig. 3B). The C6 mutation also abolished the binding of ARP-1 to region IX (data not shown).

The function of region IX NRRE was analyzed by introducing the $\mathrm{C} 6$ mutation in a Hoxd-11/lacZ reporter gene and subsequent generation of transgenic embryos. While the 7.5-kb genomic fragment including region IX (Ns-E/lacZ; Fig. 1) mimicked quite closely the pv27-restricted expression of Hoxd-11 in paraxial mesoderm (Fig. 5A), transgenes with mutated NRRE (Ns-E/lacZ*, Fig. 1) displayed a rostral shift of their limit of expression by two prevertebrae (Fig. 5B). This anterior shift was comparable to that observed upon full deletion of region IX (Ns-H/lacZ; Fig. 2B). These results thus identified the conserved core of region IX as a regulatory element through which RAR/RXR, or other members of the nuclear receptor superfamily such as COUP-TFs, can act as repressors of Hoxd-11 expression in the pv25-26 domain. 

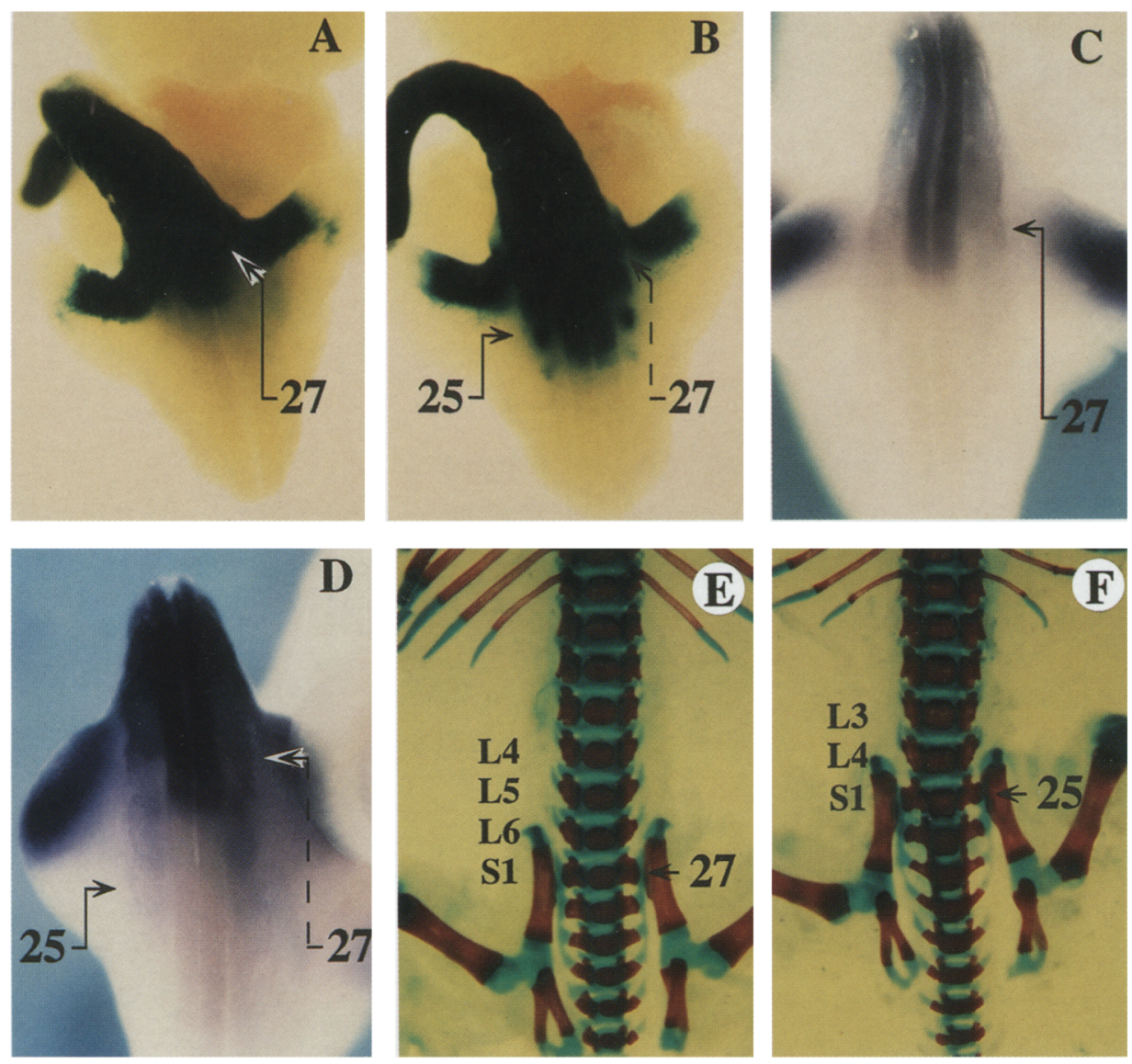

Figure 2. Expression of Hoxd-11 in prevertebrae 25-26 induces an anterior shift of the sacrum. (A) Ns-E/lac Z reporter gene expression in mid-gestation (day 11) embryos. The arrow points to the anterior limit of expression in pv27. $(B)$ In the absence of region IX (Ns-H/lacZ), the anterior limit of expression was at the level of pv25, in both spinal cord and paraxial mesoderm. $(C)$ Whole-mount in situ hybridization detection of Hoxd-11 specific transcripts in a day-10 embryo. Hoxd-11 expression boundary was at the level of pv27 (arrow). (D) Hoxd-11 expression in a Ns-H/Hoxd-11 transgenic embryo (day 10). Ectopic transcript accumulation was seen up to the level of pv25 (full arrow). (E) Ventral view of a wild-type skeleton in the lumbosacral region. The indications L3-L6 and S1 point to the different lumbar and sacral vertebrae, respectively. The L6 skeletal variant was predominant in wild-type mice and the lumbosacral transition was at the level of the 27 th vertebra. $(F)$ L4 skeletal variant in Hoxd-11 transgenic $(\mathrm{Ns}-\mathrm{H} /$ Hoxd-11) mice. The lumbosacral transition was shifted up to the level of the 25 th vertebra.

\section{Mutation of Hoxd-11 NRRE in vivo}

With the aim to analyze the function of region IX in the context of the complete $H o x D$ cluster, we introduced the C6 mutation into the resident Hoxd-11 locus via ES celltargeted mutagenesis (Fig. 4). We made use of the loxP/ cre system to subsequently remove the selectable marker (PGKneo), in order to prevent transcriptional interference (Fig. 4). All mice homozygous for this mutation showed an L5 vertebral type instead of the predominant $\mathrm{L} 6$ type found in control littermates (Fig. 5G, $\mathrm{H}$; Table 2). The L6 configuration was never observed in homozygous C6 mutants. This phenotype was codominant, for about half of the heterozygous mice showed a similar alteration, some of them with intermediate forms (Table 2). Thus, replacement of 7 nucleotides within the NRRE of region IX was sufficient to induce an anterior shift of the sacrum.
To document the effect of the mutation on the transcription of the resident Hoxd-11 gene, we analyzed the expression of Hoxd-11 in C6 mutant homozygous em-

Table 1. Number of lumbar vertebrae in Ns-H/Hoxd-11 transgenic mice $(\mathrm{Tg})$

\begin{tabular}{lcc}
\hline & \multicolumn{2}{c}{ Transgene genotype } \\
\cline { 2 - 3 } Vertebral formulae & $\operatorname{Tg}(\mathrm{n}=30)$ & control $(\mathrm{n}=15)$ \\
\hline L4 & $11^{\mathrm{a}}$ & - \\
L5 & 19 & 2 \\
L6 & - & $13^{\mathrm{b}}$ \\
\hline
\end{tabular}

All control animals included in this table were littermates of the transgenic specimen.

ancluding one animal with mixed lumbo-sacral L5.

'Including four animals with mixed lumbo-sacral L6. 


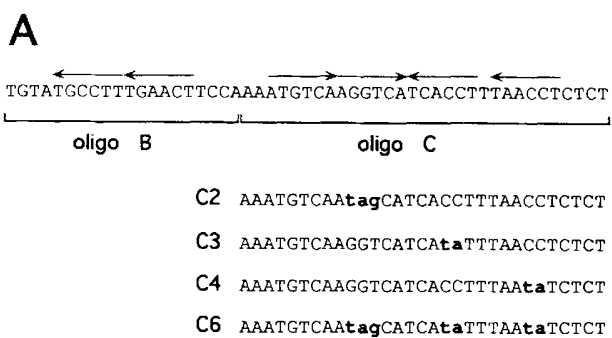

B

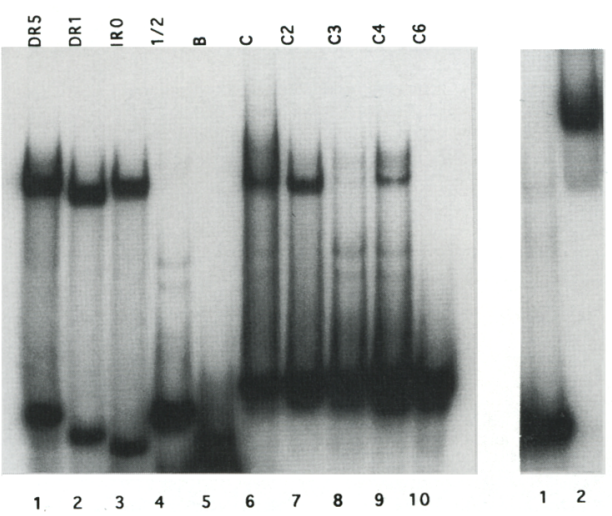

Figure 3. In vitro binding of nuclear receptors to Hoxd-11 region IX. (A) Sequence of the region IX-derived oligonucleotides $\mathrm{B}$ and $\mathrm{C}$ and oligonucleotides with mutations (lowercase, bold). Half-site motifs and their orientations are indicated by arrows on top of the sequences. $(B)$ Binding of RAR/RXR heterodimers. EMSAs with the oligonucleotide probes depicted in $A$ (lanes 5-10) and purified E. coli-expressed RAR $\alpha(25 \mathrm{ng})$ and RXR $\alpha \mathrm{DAB}$ (25 ng; Chen et al. 1994). EMSAs with the same amount of RAR $\alpha$ and RXR $\alpha$ DAB and the canonical DR5 RARE (lane 1), IR0 (lane 3) or a single half-site (1/2) element (lane 4; 5'-AGGTCA-3' motif) are shown for comparison. Lane 2 corresponds to an EMSA with a DR 1 element and $25 \mathrm{ng}$ RXR $\alpha$ DAB. (C) Binding of ARP-1 to oligo C. EMSA was performed with an extract of COS cells expressing ARP-1 (lane 2), and the same amount of COS cell mock extract (lane 1).

bryos by whole mount in situ hybridization. As predicted by the transgenic analysis, Hoxd-11 expression in C6 mutant embryos was anteriorized in the pv25-26 domain (Fig. 5, cf. C and D). Because the NRRE was located between Hoxd-11 and Hoxd-10, we wanted to detect any possible influence of the $\mathrm{C} 6$ mutation on the expression of Hoxd-10 as well. Whole mount in situ hybridization experiments with the corresponding antisense RNA probe revealed that the expression boundary of Hoxd-10 was also anteriorized in C6 mutant embryos. In the wildtype specimen, the Hoxd-10 anterior boundary was at the level of pv25 (Fig. 5E), whereas in C6 mutant embryos it was shifted unambiguously to pv24 (Fig. 5F). Therefore, both Hoxd-10 and Hoxd-11 acquired novel, but distinct, expression boundaries after in vivo mutagenesis of the NRRE. Since the newly established expression level of Hoxd-10 was more anterior than that of Hoxd-11, in C6 mutant mice, it appeared unlikely that the anterior shift of Hoxd-10 resulted from a secondary effect of Hoxd-11 anteriorization. However, to further exclude this possibility, we looked at Hoxd-10 expression in embryos transgenic for the Ns-H/Hoxd-11 construct (embryos that expressed Hoxd-11 in the pv25-26 domain). In such transgenic embryos, Hoxd-10 was expressed exactly as in wild type animals (pv25; Fig. 5E), thereby confirming that Hoxd-11 anteriorization was not the cause of the anterior shift in Hoxd-10 expression. From this, we concluded that the $\mathrm{C} 6$ regulatory mutation led to misexpression of both Hoxd-11 and Hoxd-10 through a direct cis-acting control mechanism.

Even though Hoxd-11 has an important function in

A

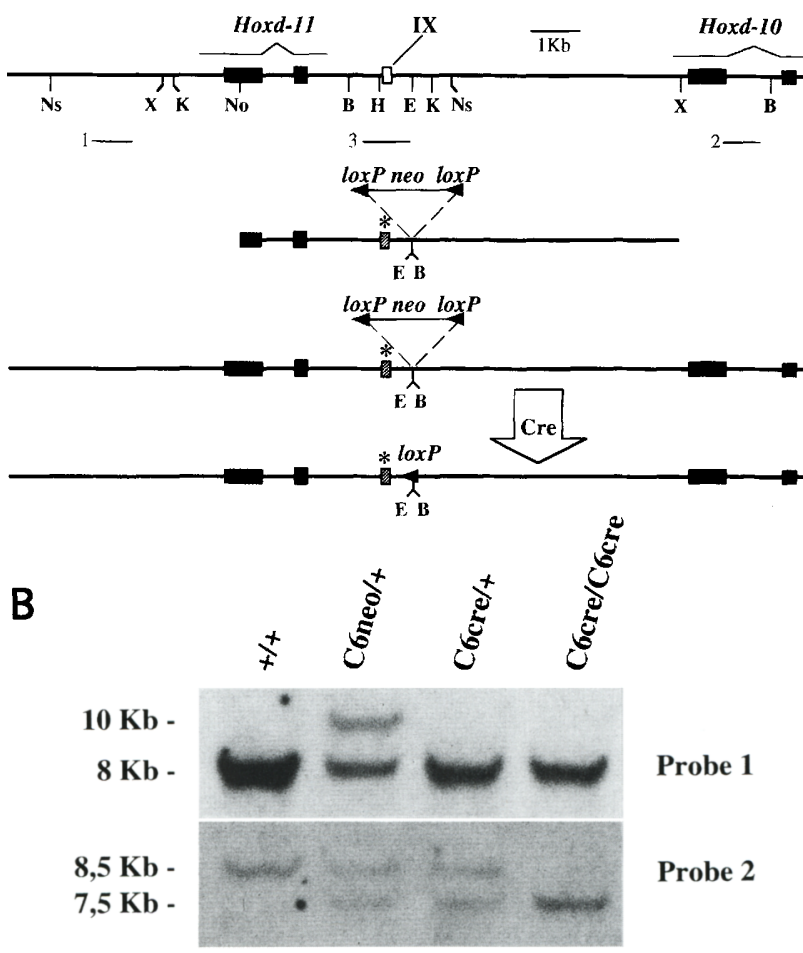

Figure 4. Introduction of the $\mathrm{C} 6$ mutation into region IX by homologous recombination in ES cells. $(A)$ Restriction map of the Hoxd-11 locus. The targeting construct (below) contained 3 $\mathrm{kb}$ of a $5^{\prime}$ homology region, the $\mathrm{C} 6$ mutation in region $\mathrm{IX}\left({ }^{*}\right), \mathrm{a}$ lox $P$-PGKneo-lox $P$ cassette in the reverse transcriptional orientation with respect to the Hoxd-11 gene, followed by $5.5 \mathrm{~kb}$ of $3^{\prime}$ homology. Below, a map of the recombined locus after homologous recombination. After treatment of ES cells with the Cre recombinase, the final configuration of the locus was obtained (bottom). (B) Southern blot detection of the homologous recombination event in ES cells and genotypes of C6 mutant animals. C6neo and C6cre indicate the mutant alleles before and after the treatment with the recombinase, respectively. Probe 1 was used to reveal NsiI digested fragments $18-\mathrm{kb}$ fragment for wild-type and C6cre alleles, $10 \mathrm{~kb}$ for the C6neo allele), and probe 2 for Bam $H$ digestions $(8.5 \mathrm{~kb}$ for the wild-type allele, $7.5 \mathrm{~kb}$ for the C6neo and C6cre alleles). KpnI and XhoI digestions were also used, with probe 3 to check for the absence of randomly integrated targeted vectors. (Ns) NsiI; (X) XhoI; (K) KpnI; (No) NotI; (B) BamHI; (H) HindIII; (E) EcoRI. 

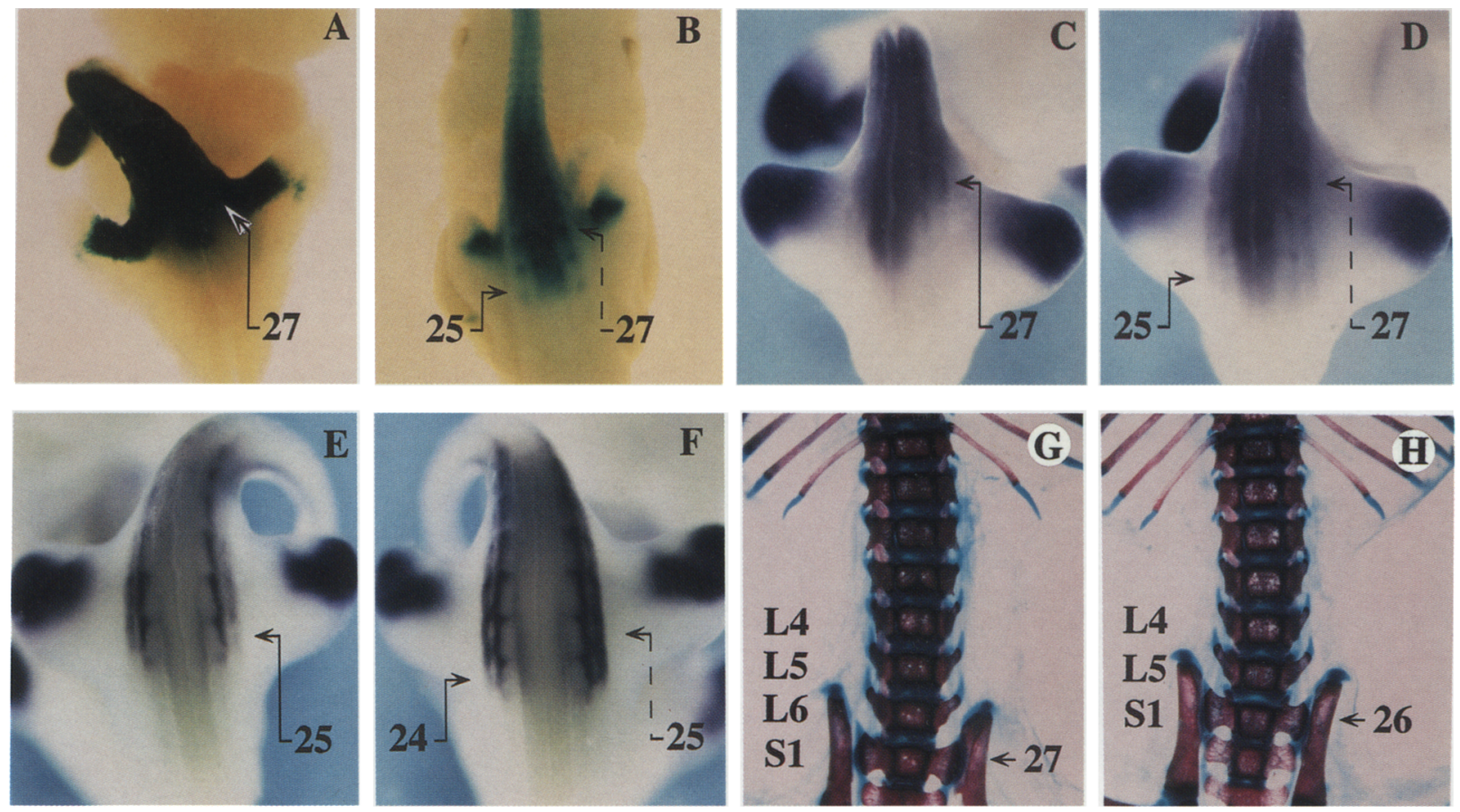

Figure 5. Anteriorized Hoxd-11 and Hoxd-10 expression in $\mathrm{C} 6$ mutant embryos $(A)$ Hoxd-11/lac $Z$ reporter gene expression in midgestation embryos established an anterior limit at the level of pv27 (Ns-E/lacZ construct). (B) Introduction of the C6 mutation in region IX NRRE (Ns-E/lacZ* construct) led to an anterior extension of lacZ expression with a novel boundary at the level of pv25 (full arrow). (C) Hoxd-11 mRNA accumulation in wild-type embryos as detected by whole-mount mRNA in situ hybridization. The arrow points to the expression boundary at the level of pv27. (D) In homozygous C6 mutant embryos, Hoxd-11 expression was shifted anteriorly in pv25-26. $(E)$ Whole-mount in situ hybridization of Hoxd-10 mRNA in wild-type embryos. Hoxd-10 expression boundary was at the level of pv25 (arrow). (F) Hoxd-10 expression was anteriorized in homozygous C6 mutant embryos, with a new boundary in pv24 (full arrow). The dashed arrow points to the wild-type Hoxd-10 boundary. ( $G$ ) Predominant L6 skeletal variant in wild-type mice. $(H)$ All mice homozygous for the C6 mutation had five lumbar vertebrae (L5 type). The indications L4-L6 and S1 denote the different lumbar and sacral vertebrae, respectively.

limbs (Davis et al. 1995), animals homozygous for the C6 mutation displayed no alteration in the appendicular skeleton, suggesting that Hoxd gene expression in developing limbs was not affected by the C6 mutation. Furthermore, we could not detect any genetic interactions by looking at the progenies of crosses involving the $\mathrm{C} 6$ allele and either Hoxd-11, Hoxa-11, or Hoxd-13 null mutant alleles, which are known to affect limb development (Dollé et al. 1993; Small and Potter 1993; Favier et

Table 2. Number of lumbar vertebrae in C6 mutant mice

\begin{tabular}{lccc}
\hline & \multicolumn{3}{c}{ C6 mutant genotype } \\
\cline { 2 - 4 } Vertebral formulae & $\begin{array}{c}\text { C6/C6 } \\
(n=20)\end{array}$ & $\begin{array}{c}\text { C6 } /+ \\
(n=25)\end{array}$ & $\begin{array}{c}+/+ \\
(n=23)\end{array}$ \\
\hline L5 & 20 & 8 & - \\
L6 & - & $17^{\mathrm{a}}$ & $23^{\mathrm{a}}$
\end{tabular}

All wild-type animals included in this table were littermates of the mutant specimen.

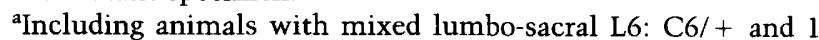
$+1+$. al. 1995; data not shown). The analysis of Hoxd-10, Hoxd-11, and Hoxd-12 expression in the developing limbs of C6 mutant and wild-type embryos did not reveal any difference (data not shown), thus confirming that the integrity of region IX NRRE is required only for Hox gene regulation in the developing trunk.

\section{Discussion}

\section{A silencer element determines the position of the sacrum}

By using a conventional transgenic approach together with interspecies DNA sequence comparison, we have identified a cis-acting element (region IX) that is necessary to position the Hoxd-11 boundary in prevertebrae. This element is able to silence Hoxd-11 expression in the pv25-26 domain but it allows its expression posteriorly. The 25 th and 26 th vertebrae correspond to the fifth and sixth lumbar vertebrae, respectively, in wildtype animals. When Hoxd-11 was expressed ectopically in the pv25-26 domain (Ns-H/Hoxd-11 transgene), vertebrae 26 and 25 (the latter in one-third of the animals) were transformed into sacral vertebrae. This result is in 
agreement with a function of Hoxd-11 in setting up the level of the lumbosacral transition at vertebra 27 , as suggested by the frequently observed shift of the first sacral vertebra from vertebra 27 to 28 in Hoxd-11 mutant mice (Davis and Capecchi 1994; Favier et al. 1995). Repression of Hoxd-11 transcription by region IX is, therefore, an important event in the molecular mechanism that establishes the level of the lumbosacral transition.

Sequence comparisons revealed that region IX contains conserved motifs related to the consensus nuclear receptor binding half-site PuGGTCA sequence. These motifs, organized as a IR0, overlapping with a DR1, were able to bind in vitro RAR/RXR heterodimers, as well as ARP-1, a member of the COUP-TF family. We refer to this DNA sequence as an NRRE, as we cannot exclude the possibility that other members of the nuclear receptor superfamily act on this element in vivo. The structure of this NRRE was conserved highly throughout vertebrate evolution, and a 7-bp mutation within this NRRE (C6 mutation) that was shown to abolish the binding of nuclear receptors led to anteriorized Hoxd-11 expression in transgenic mice. Therefore, region IX silencing activity depends upon the integrity of the NRRE. The molecular mechanism involved in this transcriptional repression in pv25 and 26 is unclear, as, for instance, $\operatorname{RAR} \alpha, \operatorname{RAR} \gamma, \operatorname{RXR} \alpha$, and $\operatorname{RXR} \beta$ are all expressed in the domains where Hoxd-11 is either repressed (pv2526) or expressed (posterior to pv26; Ruberte et al. 1990, 1991; Dollé et al. 1994). Furthermore, analyses of mice mutated for RARs have failed to reveal obvious vertebral alterations in the lumbosacral region (Lohnes et al. 1994). Members of the COUP-TF family may be good candidates for mediating the silencing activity of region IX. First, these factors are potent dominant repressors of both basal transcription and transactivation by several nuclear receptors (Mangelsdorf and Evans 1995). Second, they bind preferentially to DR1 elements and, with a lower affinity, to IR0 elements (Cooney et al. 1992). Three members of the COUP-TF family have been cloned in mice so far: COUP-TF1, ARP-1, and EAR-2. These nuclear receptors were shown to be expressed during mouse embryogenesis (Jonk et al. 1994). Analysis of both the vertebral configurations at the lumbosacral level as well as Hox expression in mice mutant for these genes will be informative regarding the possible role of this gene family in region IX-mediated silencing activity.

\section{Function of region IX in situ}

To investigate the properties of this potential NRRE under physiological conditions, that is, in the context of the clustered organization of Hox genes, we introduced the C6 mutation in the HoxD complex via homologous recombination in ES cells. We made use of the loxP/cre system (Gu et al. 1993) to excise subsequently the PGKneo selection cassette so that no transcriptional interferences with neighboring genes would be expected (Olson et al. 1996). Animals carrying the C6 mutant allele had a wrongly positioned lumbosacral transition. All homozygous specimen had five lumbar (L5) vertebrae instead of the six (L6) found in wild type. This L5 phenotype was codominant, as about half of the heterozygous mice showed a similar alteration. In contrast to animals transgenic for the Ns-H/Hoxd-11 construct, we never observed an L4 phenotype in the C6 mutant mouse line. This difference may be due to different amounts of Hoxd-11 mRNA expressed in the pv25 domain in transgenic and C6 mutant embryos.

Mutant animals were fertile and showed no abnormal phenotype in their limbs. Furthermore, the C6 allele did not interact with other Hox mutant alleles known to affect limb development. Consistently, posterior Hoxd gene expression was not affected in developing limbs of mutant embryos. Therefore, we rule out the possibility that Hoxd genes are regulated in limbs via the NRRE of region IX. We believe that these results illustrate the existence of regulatory pathways for Hoxd gene expression that are different for limbs and trunk. It also suggests how innovations in morphologies may arise due to subtle alterations in the regulation of Hox gene expression. Such variations may thus involve only a subset of the regulatory circuitry, thereby allowing more flexibility in the acquisition or modification of functions.

\section{Sharing a regulatory element}

Consistent with the results obtained in transgenic mice, the C6 mutation led to the anteriorization of Hoxd-11 expression in the pv25-26 domain. Strikingly, Hoxd-10 expression was also anteriorized in C6 mutant embryos. The possibility that this shift in Hoxd-10 expression from pv25 to pv24 was due to Hoxd-11 anteriorization was ruled out by the analysis of Hoxd-10 expression in embryos transgenic for the Ns-H/Hoxd-11 construct. Although this transgenic line expressed Hoxd-11 in the pv25-26 domain, Hoxd-10 was not anteriorized. Therefore, the NRRE in region IX is required in cis for proper expression of both Hoxd-11 and Hoxd-10, likely through a negative regulation of the two neighboring promoters (Fig. 6). However, it is as yet unclear whether Hoxd-10 is involved in positioning the sacrum as well. As this important patterning event depends, in a quantitative manner, upon the function of several $A b d B$-like genes (Zákány et al. 1996), including from paralogy group 10 (Favier et al. 1996), it is conceivable that Hoxd-10 gainof-function detected in the C6 mutant animals contributes to the anterior shift of the sacrum. The description of the Hoxd-10 mutant phenotype will be indicative in this respect.

Our results show that regulatory elements are shared between several genes within a Hox complex (Krumlauf 1994; Van der Hoeven et al. 1996), and provide evidence for both the existence of such elements in the HoxD complex and their potential, as regulatory constraints, to maintain the clustered organization. Different evolutionary schemes can account for the emergence of shared regulatory elements such as region IX. For example, it may derive from a duplication event involving an Hox gene but not a nearby pre-existing regulatory element. This element would then be recruited to regulate both 


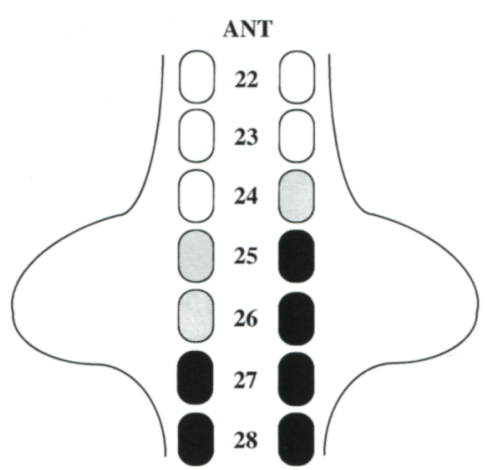

POST

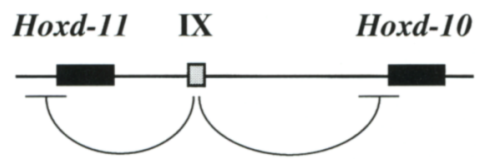

Figure 6. Region IX defines Hoxd-11 and Hoxd-10 expression boundaries. Schematic representation of a day-11 embryo at the level of the hindlimb buds. Hoxd-11 (left) and Hoxd-10 (right) expression boundaries in prevertebrae are indicated in wild-type (black) and C6 regulatory mutant (gray) embryos. Region IX, a silencer element, regulates in cis the expression of both Hoxd11 and Hoxd-10.

the original as well as the newly duplicated gene. Alternatively, the regulatory element may appear after duplication and be used further to refine the regulation of several genes. An interesting property of region IX lies in its ability to establish different AP boundaries for Hoxd11 and Hoxd-10. Indeed, region IX negatively affects Hoxd-11 in pv25-26, whereas Hoxd-10 is expressed in this domain and silenced only in pv24 (Fig. 6). Consequently, in pv25-26, the same regulatory element can be either permissive or restrictive, depending on the target promoter. This observation illustrates the functional flexibility of this shared element and might explain why such a regulatory strategy has arisen, rather than one relying exclusively on several independent gene-specific elements.

\section{Materials and methods}

\section{DNA constructs}

Ns-E/lacZ and Ns-H/lacZ constructs were described previously (Gérard et al. 1993). The Ns-H/Hoxd-11 construct was like the $\mathrm{Ns}-\mathrm{H} /$ lacZ construct, but without reporter gene sequences inserted in the Hoxd-11 coding sequence. The Ns-E/lacZ* construct was identical to the Ns-E/lacZ construct, except for the $\mathrm{C} 6$ mutation which was introduced in region IX by PCR. The PCR fragment containing the $\mathrm{C} 6$ mutation was verified by sequencing before cloning into the Ns-E/lacZ* construct. For injection, all fragments were excised with NsiI. For the construction of the targeting vector for homologous recombination, the loxP-PGKneo-loxP cassette was cloned $5^{\prime}$ to the $5.5 \mathrm{~kb} E c o R \mathrm{I}-$ $X$ hoI fragment from the Hoxd-11/Hoxd-10 intergenic region. The 3.5-kb NotI-EcoRI fragment from Ns-E/lacZ $Z^{\star}$, containing the C6 mutation, was then cloned $5^{\prime}$ to the IoxP-PGKneo-loxP cassette. This construct was linearized with XhoI prior to electroporation into ES cells.

\section{Transgenic mice}

Transgenic mice were obtained by DNA microinjection into C57BL/6xCBA F1 eggs. Transgenic embryos were analyzed by whole-mount enzyme histochemical detection of $\beta$-gal activity (Zákány et al. 1988). Seven out of 10 transgenic fetuses expressed the Ns-H/lacZ construct, and the pv27 restricted expression of the Ns-E/lacZ was seen in 11 out of 12 independent integrants (Gérard et al. 1993). Three embryos at day 11 that all showed anteriorized expression in the pv25-26 domains were obtained with the Ns-E/lac $Z^{\star}$ construct (Fig. 5). Five transgenic families were generated with the Ns-H/Hoxd-11 fragment. Two families showed vertebral alterations of the L4 type ; one of these displayed the L4 phenotype only in homozygous animals (J. Zákány, M. Gérard, D. Duboule, unpubl.). The differences in expressivity between the two transgenic families were likely due to different levels of Hoxd-11 expression in pv25 and pv26. Both in L4 and L5 animals, vertebrae anterior to L3 remained unchanged. In most transgenic animals, the posterolateral accessory process on the neural arch of L3 was either reduced or absent.

\section{ES cells and microinjection}

D3 ES cells (Doetschman et al. 1985) were cultured on embryonic fibroblasts in presence of $\sim 1000 \mathrm{U} / \mathrm{ml}$ LIF produced by transfection of the expression plasmid pC10-6R DIA/LIF. $2 \times 10^{7}$ ES cells were electroporated with $40 \mu \mathrm{g}$ of linearized DNA (Biorad Gene PulserTM, $400 \mathrm{~V}, 125 \mu \mathrm{F}, 2.3 \mathrm{~s}$ ). Cells were selected and amplified according to standard procedures (Joyner 1993). Of the clones resistant to G418, $6 \%$ had properly recombined the targeted vector. The loxP-PGKneo-lox $P$ cassette was removed from the targeted chromosome by electroporation of a CRE-protein expressing plasmid (pMC-Cre; Gu et al. 1993). Microinjection of ES cells into C57BL/ 6 blastocysts and recovering of germ-line transmission were carried out following standard procedures. Chimeric males were mated to C57BL/ 6 females, and F1 agouti progeny was genotyped.

\section{Genotype analysis}

The presence of the C6 mutation in region IX was verified by PCR amplification of region IX, from both ES cell and F1 animal genomic DNA, by using a primer $5^{\prime}$ to region IX $\left(5^{\prime}\right.$ AGAAGCCCAAAGTCTAAAAATAA-3'), and a $3^{\prime}$ primer derived from the lox $P$ sequence $\left(5^{\prime}\right.$-ATAACTTCGTATAATGTATGCTAT$\left.3^{\prime}\right)$. PCR fragments $(850 \mathrm{bp})$ were subcloned and sequenced. The C6 mutant allele was routinely identified by Southern blot analysis on tail or yolk sac genomic DNA using the probes depicted in Figure 4: probes 1 (HindIII-BgIII 780-bp fragment), 2 (PstI 830-bp fragment), and 3 (XbaI-EcoRI 910-bp fragment). The presence of the Ns-H/Hoxd-11 transgene was followed on Southern blots of genomic DNA, probed with Hoxd-11 PstIHindIII 330-bp region VIII fragment (Gérard et al. 1993), detecting a transgene specific 800-bp BamHI fragment.

\section{Electrophoretic Mobility Shift Assays}

EMSAs were performed as described in Mader et al. (1993) and Chen et al. (1994). ARP-1 was expressed in COS cells under the control of the SV40 promoter in the pSG5 expression vector (Green et al. 1988). 


\section{Whole-mount in situ hybridization and skeletal analysis}

Whole-mount in situ hybridizations were performed according to established procedures. Antisense Hoxd-11 RNA probe was transcribed from a 777-bp Hoxd-11 cDNA (Gérard et al. 1993). Antisense Hoxd-10 RNA probe was transcribed from a 1138-bp Hoxd-10 cDNA PCR fragment (Renucci et al. 1992). Skeletal preparations were made at the age of one or six days after birth and stained with the alizarin red and alcian blue double-staining protocol (Inouye 1976).

\section{Acknowledgments}

We thank Drs. R. Kemler, K. Rajewski, A. Smith, and P. Kaestner for the gift of the ES cells, plasmids expressing Cre, LIF, and the PGKneo cassette, respectively. We thank M. Friedly for invaluable help with ES cells, the members of the Duboule laboratory for helpful comments and discussions, as well as Dan Lavery for suggestions on the manuscript. J.Z. was on leave from the Institute of Genetics of the Biological Research Center of the Hungarian Academy of Sciences, Szeged. M.G. was the recipient of fellowships from the Roche Research Foundation and the Communaute Economique Europeenne. This work was supported by funds from the Fond National Suisse de la Recherche Scientifique, the Canton de Genève, the Claraz Foundation, the Latsis Foundation and the Human Frontier Science Program Organization (Geneva), and from the Institut National de la Santé et la Recherche Médicale, the Centre National de la Recherche Scientifique, the Association pour la Recherche contre le Cancer, and Foundation pour la Recherche Médicale (Illkirch).

The publication costs of this article were defrayed in part by payment of page charges. This article must therefore be hereby marked "advertisement" in accordance with 18 USC section 1734 solely to indicate this fact.

\section{References}

Capecchi, M.R. 1989. Altering the genome by homologous recombination. Science 244: 1288-1292.

Chen, Z.P., L. Shemshedini, B. Durand, N. Noy, P. Chambon, and H. Gronemeyer. 1994. Pure and functionally homogeneous recombinant retinoid $\mathrm{X}$ receptor. I. Biol. Chem. 269: 25770-25776.

Cooney, A.J., S.Y. Tsai, B.W. O'Malley, and M.Y. Tsai. 1992. Chicken ovalbumin upstream promoter transcription factor (COUP-TF) dimers bind to different GGTCA response elements, allowing COUP-TF to repress hormonal induction of the vitamin D3, thyroid hormone, and retinoic acid receptors. Mol. Cell. Biol. 12: 4153-4163.

Davis, A.P. and M.R. Capecchi. 1994. Axial homeosis and appendicular skeleton defects in mice with a targeted disruption of Hoxd-11. Development 120: 2187-2198.

Davis, A.P., D.P. Witte, H.M. Hsieh-Li, S.S. Potter, and M. Capecchi. 1995. Absence of radius and ulna in mice lacking hoxa-11 and hoxd-11. Nature 375: 791-795.

Doetschman, T.C., H. Eistetter, M. Katz, W. Schmidt, and R. Kemler. 1985. The in vitro development of blastocyst-derived embryonic stem cell lines: Formation of visceral yolk sac, blood islands and myocardium. J. Embryol. Exp. Morpholol. 87: 27-45.

Dollé, P., A. Dierich, M. Le Meur, T. Schimmang, B. Schuhbaur, P. Chambon, and D. Duboule. 1993. Disruption of the Hoxd13 gene induces localized heterochrony leading to mice with neotenic limbs. Cell 75: 43-1-441.
Dollé, P., V. Fraulob, P. Kastner, and P. Chambon. 1994. Developmental expression of murine retinoid $X$ receptor $(R X R$ ) genes. Mech. Dev. 45: 91-104.

Favier, B., M. LeMeur, P. Chambon, and P. Dollé. 1995. Axial skeleton homeosis and forelimb malformations in Hoxd-11 mutant mice. Proc. Natl. Acad. Sci. 92: 310-314.

Favier, B., F. Rijli, C. Fromental-Ramain, V. Fraulob, P. Chambon, and P. Dollé. 1996. Functional cooperation between the non-paralogous genes Hoxa-10 and Hoxd-11 in the developing forelimb and axial skeleton. Development 122: 449-460.

Gérard, M., D. Duboule, and J. Zákány. 1993. Structure and activity of regulatory elements involved in the activation of the Hoxd-11 gene during late gastrulation. $E M B O J$. 12: 3539-3550.

Green, S., I. Issemann, and E. Sheer. 1988. A versatile in vivo and in vitro eukaryotic expression vector for protein engineering. Nucleic Acids Res. 16: 369.

Gu, H., Y.R. Zhou, and K. Rajewsky. 1993. Independent control of immunoglobulin switch recombination at individual switch regions evidenced through Cre-loxP-mediated gene targeting. Cell 73: 1155-1164.

Holland, P. 1992. Homeobox genes in vertebrate evolution. Bioessays 14: 267-273.

Inouye, M. 1976. Differential staining of cartilage and bone in fetal mouse skeleton by alcian blue and alizarin red. Congenital Anom. 16: 171-173.

Izpisúa-Belmonte, J.-C., H. Falkenstein, P. Dollé, A. Renucci, and D. Duboule. 1991. Murine genes related to the Drosophila $A b d B$ homeotic genes are sequentially expressed during development of the posterior part of the body. EMBO $/$. 10: 2279-2289.

Jonk, L.J.C., M.E.J. de Jonge, C.E.G.M. Pals, S. Wissink, J.M.A. Vervaart, J. Schoorlemmer, and W. Kruijer. 1994. Cloning and expression during development of three murine members of the COUP family of nuclear orphan receptors. Mech. Dev. 47: 81-97.

Joyner, A.L. 1993 Gene targeting. A practical approach. IRL Press at Oxford University Press, Oxford, UK.

Kessel, M. and P. Gruss. 1991. Homeotic transformation of murine vertebrae and concommitant alteration of Hox code induced by retinoic acid. Cell 67: 89-104.

Krumlauf, R. 1994. Hox genes in vertebrate development. Cell 78: 191-201.

Ladias, J.A.A. and S.N. Karathanasis. 1991. Regulation of the apolipoprotein AI gene by ARP-1, a novel member of the steroid receptor superfamily. Science 251: 561-565.

Leid, M., P. Kastner, and P. Chambon. 1992. Multiplicity generates diversity in the retinoic acid signalling pathways. Trends Biochem. Sci. 17: 427-433.

Lohnes, D., M. Mark, C. Mendelsohn, P. Dollé, A. Dierich, P. Gorry, A. Gansmuller, and P. Chambon. 1994. Function of the retinoic acid receptors (RARs) during development. (I) Craniofacial and skeletal abnormalities in RAR double mutants. Development 120: 2723-2748.

Mader, S., J.Y. Chen, Z.P. Chen, J. White, P. Chambon, and H. Gronemeyer. 1993. The patterns of binding of RAR, RXR and TR homo- and heterodimers to direct repeats are dictated by the binding specificites of the DNA binding domains. EMBO I. 12: 5029-5041.

Mangelsdorf, D.J. and R.M. Evans. 1995. The RXR heterodimers and orphan receptors. Cell 83: 841-850.

Olson, E.N., H. H. Arnold, P. W. J. Rigby, and B. J. Wold. 1996. Know your neighbors: Three phenotypes in null mutants of the myogenic bHLH gene MRF4. Cell 85: 1-4.

Renucci, A., V. Zappavigna, J. Zákány, J.C. Izpisúa-Belmonte, K. Bürki, and D. Duboule. 1992. Comparison of mouse and hu- 
Gérard et al.

man HOX-4 complexes defines conserved sequences involved in the regulation of Hox-4.4. EMBO I. 11: 1459-1468.

Ruberte, E., P. Dollé, A. Krust, A. Zelent, G. Morris-Kay, and P. Chambon. 1990. Specific spatial and temporal distribution of retinoic acid receptor gamma transcripts during mouse embryogenesis. Development 108: 213-222.

Ruberte, E., P. Dollé, P. Chambon, and G. Morris-Kay. 1991 Retinoic acid receptors and cellular retinoid binding proteins. II. Their differential pattern of transcription during early morphogenesis in mouse embryos. Development 111: $45-60$.

Small, K.M. and S.S. Potter, 1993. Homeotic transformations and limb defects in Hoxa-11 mutant mice. Genes \& Dev. 7: 2318-2328.

Van der Hoeven, F., J. Zákány, and D. Duboule. 1996. Gene transpositions in the $\operatorname{Hox} D$ complex reveal a hierarchy of regulatory controls. Cell 85: 1025-1035.

Zákány, J., C.K. Tuggle, M. Patel, and M.C. Nguyen-Huu. 1988. Spatial regulation of homeobox gene fusions in the embryonic central nervous system of transgenic mice. Neuron 1: 679-691.

Zákány, J., M. Gérard, B. Favier, S.S. Potter, and D. Duboule. 1996. Functional equivalenve and rescue among group 11 Hox gene products in vertebral patterning. Dev. Biol. 176: 325-328. 


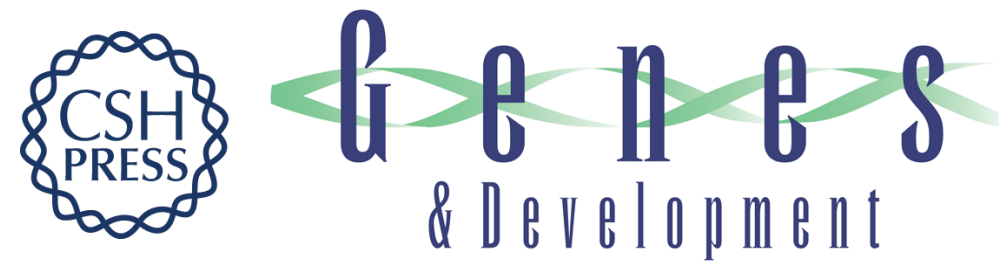

\section{In vivo targeted mutagenesis of a regulatory element required for positioning the Hoxd-11 and Hoxd-10 expression boundaries.}

M Gérard, J Y Chen, H Gronemeyer, et al.

Genes Dev. 1996, 10:

Access the most recent version at doi:10.1101/gad.10.18.2326

References This article cites 31 articles, 11 of which can be accessed free at:

http://genesdev.cshlp.org/content/10/18/2326.full.html\#ref-list-1

License

Email Alerting

Service

Receive free email alerts when new articles cite this article - sign up in the box at the top right corner of the article or click here.

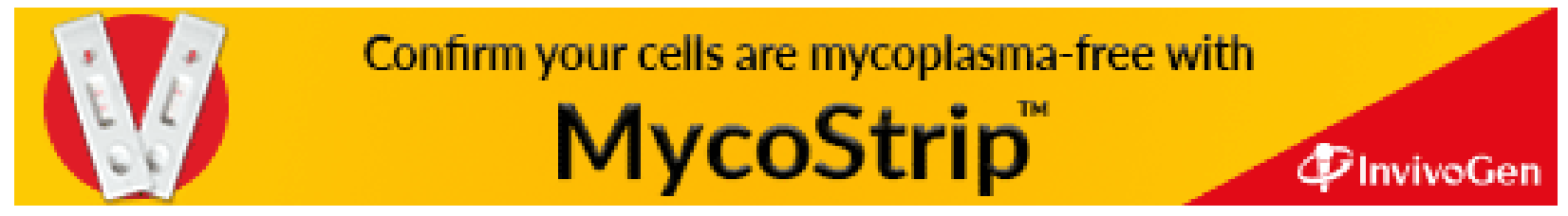

